\title{
Membrane Organization and Lipid Rafts
}

\author{
Kai Simons and Julio L. Sampaio \\ Max Planck Institute of Molecular Cell Biology and Genetics, 01307 Dresden, Germany \\ Correspondence: simons@mpi-cbg.de
}

Cell membranes are composed of a lipid bilayer, containing proteins that span the bilayer and/or interact with the lipids on either side of the two leaflets. Although recent advances in lipid analytics show that membranes in eukaryotic cells contain hundreds of different lipid species, the function of this lipid diversity remains enigmatic. The basic structure of cell membranes is the lipid bilayer, composed of two apposing leaflets, forming a twodimensional liquid with fascinating properties designed to perform the functions cells require. To coordinate these functions, the bilayer has evolved the propensity to segregate its constituents laterally. This capability is based on dynamic liquid-liquid immiscibility and underlies the raft concept of membrane subcompartmentalization. This principle combines the potential for sphingolipid-cholesterol self-assembly with protein specificity to focus and regulate membrane bioactivity. Here we will review the emerging principles of membrane architecture with special emphasis on lipid organization and domain formation.

\begin{abstract}
$A^{1 l}$ cells are delimited by membranes, which Aconfer them spatial identity and define the boundary between intracellular and extracellular space. These membranes are composed of lipids and proteins. The propensity of the hydrophobic moieties of lipids to self-associate and the tendency of the hydrophilic moieties to interact with aqueous environments and with each other is the physical basis of the spontaneous formation of the lipid bilayer of cell membranes. This principle of amphipathicity of lipids is the chemical property that enables the cells to segregate their internal constituents from the external environment. This same principle acts at the subcellular level to assemble the membranes surrounding each cellular organelle. About one-third of the genome encodes membrane proteins, and many other
\end{abstract}

proteins spend part of their lifetime bound to membranes. Membranes are the sites where many cellular machineries carry out their function.

This remarkable liquid with its amphipathic constituents is attracting increasing attention not only by biologists but also by physicists because of its fascinating properties. Membrane research has picked up speed in recent years. An increasing number of atomic structures of membrane proteins are being solved, the field of lipid research is exploding, and the principles of membrane organization are being overhauled. New insights into the staggering capability of cell membranes to subcompartmentalize have revealed how membranes support intracellular membrane trafficking and parallel processing of signaling events.

Editor: Kai Simons

Additional Perspectives on The Biology of Lipids available at www.cshperspectives.org

Copyright (C) 2011 Cold Spring Harbor Laboratory Press; all rights reserved; doi: 10.1101/cshperspect.a004697

Cite this article as Cold Spring Harb Perspect Biol 2011;3:a004697 
Here we will review the emerging principles of membrane architecture with special emphasis on lipid organization and domain formation.

\section{LIPIDS}

Lipids are amphipathic in nature containing a hydrophobic domain, a "water-fearing" or apolar end and a hydrophilic domain, which readily interacts with water. The basic premise of the hydrophobic effect is that the hydrocarbon domains of lipids distort the stable hydrogen bonded structure of water by inducing cage-like structures around the apolar domains. Selfassociation of the hydrophobic domains minimizes the total surface that is in contact with water resulting in an entropy-driven relaxation of water structure and an energy minimum for the self-associated molecular organization. The polar domains of lipids interact with water and other head groups and are therefore energetically stable in an aqueous environment (Vance and Vance 2008).

At physiologically relevant lipid concentrations (well above the critical micellar concentration) the hydrophobic effect and the shape of amphipathic molecules define three supramolecular structural organizations (phases) of lipids in solution (Fig. 1) (Seddon et al. 1997; Mouritsen 2005). The overall structures reflect the optimal packing of amphiphilic molecules at an energy minimum by balancing the hydrophobic effect and the repulsive force of close head group association. This ability of lipids to assemble into different structural associations is referred to as lipid polymorphisms (Fig. 1) (Frolov et al. 2011).

Schematic representations of membranes give the wrong idea that biological membranes are usually planar. Cell membranes can form very complex membranous structures, as seen in the myelin sheet or in the inner membrane of a mitochondrion. Different organelles have specific lipid compositions, which are important in determining their shape. Many of the lipids of eukaryotic cells are not cylindrical in shape so they, in theory, would not support the formation of a membrane bilayer (Mouritsen 2005). However, biological membranes

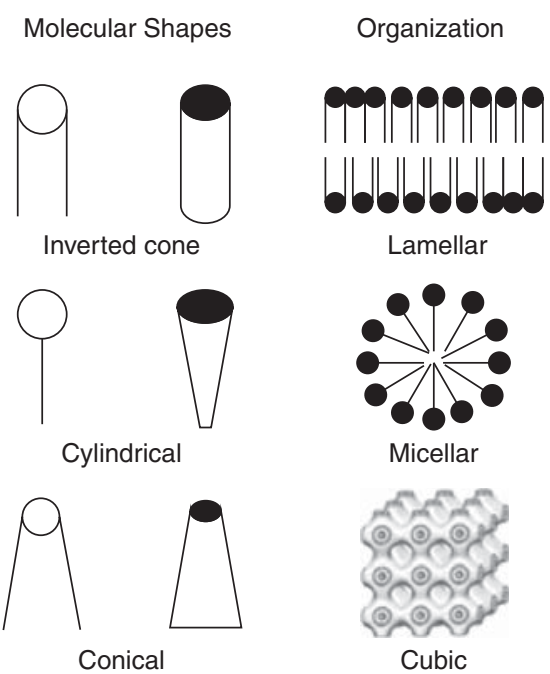

Figure 1. Lipid shape and supramolecular organization (polymorphism). Phospholipids can be classified as cylinders (e.g., PC), cones (e.g., PE), and inverted cones (e.g., lysophosphatidylcholine), depending on the relative volumes of their polar head groups and fatty acyl chains. The supramolecular organization of such molecules generates the widespread bilayer (or lamellar) structure, and the nonlamellar micellar and cubic phases.

are mostly lamellar, implying that lipids are arranged both with each other and with proteins (e.g., through selective macromolecular assemblies, specific bilayer asymmetries) to make the lamellar state more favorable.

\section{THE BILAYER}

The basic structure of all cell membranes is the lipid bilayer, the oldest still valid molecular model of cellular structures (Gorter and Grendel 1925). It is important to emphasize that cellular membranes are not dominated by the lipids but are packed with intercalated transmembrane proteins (Engelman 2005; Jacobson et al. 2007; Coskun and Simons 2010). Thus, cellular membranes are crowded two-dimensional solutions of integral membrane proteins in a lipid bilayer solvent. This fluid also has interactions with extrinsic membrane proteins. Both the membrane proteins and the lipids have bilateral compositional asymmetry, an 
important property that for the lipids consumes considerable amounts of ATP to maintain (van Meer 2011).

Although it is possible to form a lipid membrane that could act as a physical barrier from a single lipid component, the cell invested significant resources in generating a zoo of lipids to inhabit its membranes (Shevchenko and Simons 2010; Wenk 2010). Eukaryotic membrane lipids are glycerophospholipids, sphingolipids, and sterols. Mammalian cell membranes contain mainly one sterol, namely cholesterol, but a variety of hundreds of different lipid species of the first two classes. The head group of glycerophospholipids can vary, as can the bonds linking the hydrocarbon chains to glycerol, as can the fatty acids, which differ in length and degree of saturation. Also, the sphingolipids have the combinatorial propensity to create diversity by different ceramide backbones and, above all, more than 500 different carbohydrate structures, which make up the head groups of the glycosphingolipids (Futerman and Hannun 2004). Sterols were probably introduced to the lipidome later than phospholipids and sphingolipids. The advent of sterols in evolution coincided with the introduction of increasing concentrations of oxygen around 2.5 billion years ago, when eukaryotic life emerged (Mouritsen and Zuckermann 2004). Sterol synthesis requires about 30 enzymes, and the steps after generating squalene are dependent on oxygen. Eukaryotic cells spend considerable energy to synthesize this molecule that can be toxic under certain conditions, so tight mechanisms are required to regulate sterol concentrations (Ye and Debose-Boyd 2011).

The reasons for the lipid complexity are manifold. One role of the compositional diversity is to ensure a stable and robust assembly that remains impermeable, even when composition, osmolarity, or $\mathrm{pH}$ are locally changed because of physiological or pathological events. In single-component systems, slight changes in local conditions easily lead to perturbation or even disruption of the bilayer. However, these transformations are much less likely to occur in a complex system specifically designed to buffer perturbations. Lipids also have to fill the holes at the protein-lipid interfaces that result from the construction of membranespanning domains, and it is possible that lipid diversity is indeed needed to complement transmembrane domain diversity, so that membrane leakage can be prevented by exact matching.

Noteworthy is the direct correlation between membrane "architectural" sophistication and lipid diversity (Table 1). An obvious example to illustrate this principle is the comparison between prokaryotic and eukaryotic cells, the latter possessing multiple membrane compartments, the organelles. This increase in membrane morphological complexity is reflected in their lipidomes. Prokaryotic cells have only a hundred or so different lipid species, whereas eukaryotic organisms possess up to thousands. Contributing to this increase in lipid complexity in eukaryotic cells is the presence of two very important lipid categories that are exclusive to eukaryotes, sterols, and a great variety of sphingolipids. Noteworthy is the growing body of evidence that these categories are key players in membrane trafficking, a phenomenon inherently exclusive to eukaryotic cells. The preferential association between sterols, sphingolipids, and specific proteins bestows cell membranes with lateral segregation potential, which can be used for vesicular trafficking.

The increasing complexity of the cellular architecture of eukaryotic cells also raises demands on lipid functionalities. The membranes surrounding cellular organelles have different and characteristic lipid compositions. One emerging area of membrane research is the study of how different lipids interact with membrane proteins to modulate their functions (Contreras et al. 2011).

All these structural and functional features of membranes require a broad spectrum of lipid structures. Although there are a few emerging principles, this area is still in its infancy and we have a long way to go to understand the nature and consequences of lipid compositional complexity. In the next sections, we will examine how sterols and sphingolipids contribute to the organization of the biosynthetic pathway from the endoplasmic reticulum (ER) to the plasma membrane (PM). 
K. Simons and J.L. Sampaio

Table 1. Correlation between lipid compositional complexity and cellular architecture and function

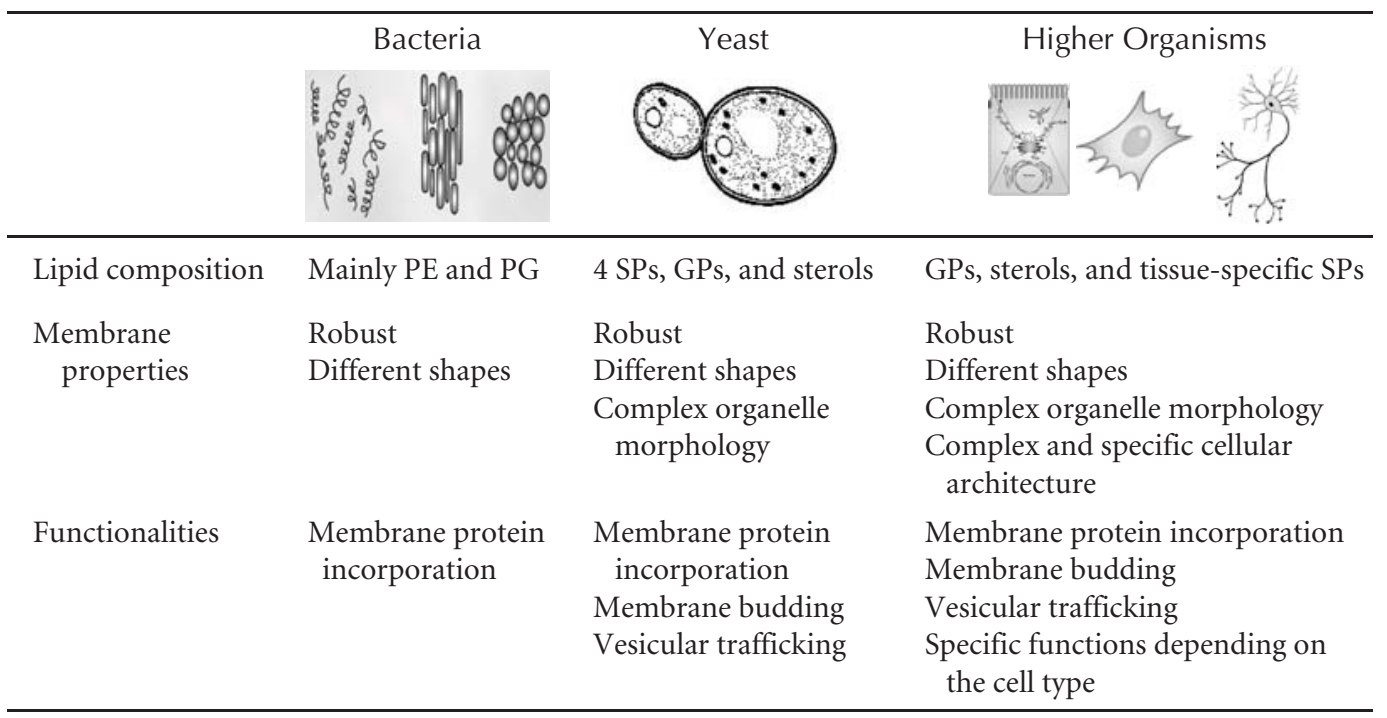

Sphingolipids (SPs) and sterols enable eukaryotic cellular membranes with the property of vesicular trafficking important for the establishment and maintenance of distinct organelles. Tissue-specific SPs in higher organisms enable the generation of specific architecture and function

\section{ROLE OF STEROLS IN BIOSYNTHETIC TRAFFIC FROM THE ER TO THE GOLGI}

One important principle in trans-membrane protein-lipid interaction is the matching of the length of the hydrophobic protein transmembrane domain (TMD) with the thickness of the lipid bilayer (Mouritsen 2011). Studies by Munro and Bretscher revealed that TMDs of plasma membrane proteins are in general longer than those of the ER and the Golgi complex (Bretscher and Munro 1993). This was recently confirmed by a large dataset from both fungi and vertebrates (Sharpe et al. 2010). The physical mechanism for increasing membrane thickness derives from the increase in sterol content from the ER (around $5 \mathrm{~mol}$ $\%$ ) to the PM, which is more than $40 \mathrm{~mol} \%$ sterol with the Golgi complex having intermediate values. Cholesterol is known to increase the thickness of lipid bilayers, but both theoretical and recent experimental studies show that it is not only bilayer thickness but also its stiffness which increases with cholesterol content, and that both of these parameters may be important for interaction with membrane proteins. By thickening and stiffening the membranes, cholesterol potentiates the intrinsic sorting of mismatched systems (Lundbæk et al. 2003). Recent experiments showed that the shorter (Golgi) TMD peptides segregated from longer (PM) TMD peptides when cholesterol concentration was increased in bilayers where fatty acid length was shorter than the length of the longer peptide. These data show experimentally that cholesterol content can induce protein sorting (HJ Kaiser, A Orlowsky, T Rog, et al. unpubl.).

Altogether, these studies suggest that the cholesterol gradient plays an important role in organizing the biosynthetic pathway. In the ER, newly synthesized proteins of various TMD lengths would be incorporated into the cholesterol-poor-therefore, more adaptable-membrane of the ER, where they would remain mixed until sorting before departure to the cis-Golgi. In the Golgi complex, cholesterol concentration increases toward the trans-side, promoting sorting of shorter Golgi proteins from longer TMD proteins, which proceed toward the PM. 


\section{ROLE OF STEROL AND SPHINGOLIPIDS IN POST-GOLGI TRAFFIC}

In the secretory pathway, sorting of not only proteins but also of lipids has to occur before exit from the trans-Golgi network. The existence of separate pathways emanating from the Golgi complex implies that hydrophobic matching and mismatching cannot be the only principle involved. It is well known that coat/adaptor-mediated sorting involves cytoplasmic determinants present in trans-membrane cargo proteins, which target specific proteins to endosomes for further delivery to their cellular destination (e.g., the basolateral plasma membrane of epithelial cells). The increasing concentration of sterols and sphingolipids is enhanced by retrograde COPI-mediated transport in the Golgi complex. Retrograde COPI transport vesicles have been shown to be depleted in cholesterol and sphingomyelin, increasing the content of sterols and sphingolipids toward the trans-side of the Golgi complex (Brugger et al. 2000).

Studies in yeast and epithelial MadinDarby canine kidney (MDCK) cells show that there are pathways from the trans-Golgi, distinct from the well-described coat-mediated routes, which show the capacity to sort membrane lipids.

Yeast

Two cell surface delivery pathways have been identified in yeast and one of these is a direct TGN to PM route (Harsay and Bretscher 1995; Gurunathan et al. 2002). This latter route has been shown to transport trans-membrane proteins that are resistant to extraction by detergent at $4^{\circ} \mathrm{C}$ (Bagnat et al. 2000). Using one such protein as a probe, yeast mutants, for sterol and sphingolipids biosynthesis were identified as critical for post-Golgi transport in a genomewide screen (Proszynski et al. 2005). These lipid mutants led to impaired exit from the TGN. Using an elaborate immuno-isolation protocol, the post-Golgi transport vesicles, carrying the same protein probe that was employed in the genetic screen, were isolated (Klemm et al. 2009). Lipidomic analysis of the purified carriers convincingly showed that sterol and all yeast sphingolipid species were dramatically enriched when compared to the isolated donor organelle. These findings unequivocally showed that lipid sorting occurs in the TGN, enhancing the enrichment of sterols and sphingolipids in the PM. Recent studies have extended these studies to other PM proteins. They are all sorted into transport carriers that are similarly enriched in sterols and sphingolipids (M Surma, C Klose, K Simons, unpubl.).

\section{MDCK Cells}

Epithelial cells also have two (at least) pathways to the cell surface (Schuck and Simons 2004; Rodriguez-Boulan et al. 2005). These are directed to the apical and basolateral PM domains, respectively. The apical membrane mediates many of the functions specific to epithelial cells. This PM domain is exposed to the external world and forms a robust barrier that protects the intestine, kidney, and other tissues against the hostilities of the outside environment. The unusual robustness of the apical membrane is largely because of its specific lipid composition. It is strongly enriched in glycosphingolipids, which together with cholesterol form a rigid membrane barrier (Kawai et al. 1974; Sampaio et al. 2010). Early evidence in epithelial MDCK cells suggested that sorting of glycosphingolipids takes place in the TGN and that these lipids are preferentially sorted to the apical membrane (Simons and Van Meer 1988). Apical membrane proteins were shown to become detergent resistant after entering the Golgi complex (Skibbens et al. 1989; Brown and Rose 1992; Fiedler et al. 1993). Moreover, decreasing cellular cholesterol led to impairment of apical protein transport whereas basolateral transport was unaffected (Keller and Simons 1998). Finally, sphingolipid integrity was also shown to be required for the apical transport machinery by inhibitor studies (Mays et al. 1995).

Recent studies have implicated a lectin, galectin-9, as a critical factor in apical membrane biogenesis (Mishra et al. 2010). When galectin-9 was knocked down by RNAi, the 
MDCK cells failed to polarize and establish apical-basolateral polarity. Importantly, this lectin was shown to be apically secreted by a mechanism that bypasses the ER and the Golgi complex (Friedrichs et al. 2007). Strikingly, when exogenous galectin-9 was introduced to depolarized MDCK cells depleted of endogenous galectin-9, the cells repolarized to form an asymmetric cell layer. The lectin was found to bind the Forssman glycolipid and became endocytosed. After reaching the TGN, the galectin recycled back to the apical membrane. This lectin-Forssman glycolipid circuit may be instrumental in maintaining apical transport in MDCK cells (Mishra et al. 2010).

The significance of the galectin-glycolipid interaction was underpinned by a comprehensive lipid analysis of the changes occurring during polarization of the MDCK cells from the contact-naive unpolarized state to the final epithelial sheet (Sampaio et al. 2010). The most striking changes occurring during polarization were that the sphingolipids became longer, more hydroxylated, and more glycosylated than their counterparts in the unpolarized cells. Conversely, the glycerolipids acquired, in general, longer but more unsaturated fatty acids. Most importantly, the Forssman glycosphingolipid was practically absent in the unpolarized MDCK cells and became the major sphingolipid in the fully polarized state. When the MDCK cells depolarized toward the mesenchymal state, the lipids changed back to that of the contact-naive cells. Thus, the finding that galectin-9 interacts with the Forssman glycolipid could be key to understanding the mechanism of protein and lipid sorting in the TGN of MDCK cells. A similar galectin-glycolipid circuit has been unveiled in epithelial HT29 cells, where galectin- 4 binds to sulfatide and becomes part of the apical sorting machinery (Delacour et al. 2005).

\section{LIPID RAFTS AS A MEMBRANE- ORGANIZING PRINCIPLE}

The lipid raft concept was introduced to explain the generation of the glycolipid-rich apical membrane of epithelial cells (Simons and Van
Meer 1988). The hypothesis matured and was later generalized as a principle of membrane subcompartmentalization, functioning not only in post-Golgi trafficking, but also in endocytosis, signaling, and many other membrane functions (Simons and Ikonen 1997). Presently, membrane rafts are defined as dynamic nanoscale sterol, sphingolipid-enriched, ordered assemblies of specific proteins, in which the metastable resting state can be activated to coalesce by specific lipid-lipid, protein-lipid, and protein-protein interactions (Hancock 2006; Lingwood and Simons 2010; Simons and Gerl 2010). The lipids in these assemblies are thought to be enriched in saturated and longer hydrocarbon chains and hydroxylated ceramide backbones.

The studies on post-Golgi membrane traffic to the PM described in the previous section conform to what would be expected for a sphingolipid/sterol-based raft transport mechanism. Yeast lipid mutants that caused impaired Golgi exit involved ergosterol and sphingolipid synthesis (Proszynski et al. 2005). The two strongest phenotypes observed in sphingolipid-related mutants affected the elongation of the ceramide fatty acid from C22 to C26 (elo3) and hydroxylation of the sphingosine moiety (sur2). These molecular attributes have been implicated not only in the formation of liquid-ordered $\mathrm{L}_{\mathrm{o}}$ phases (Heberle and Feigenson 2011; Mouritsen 2011) but also in the coupling the two membrane leaflets in the rafts by interdigitation of the very long chain fatty acid from the exoplasmic into the underlying cytoplasmic leaflet (elo3) and by augmenting hydrogen bonding in sphingolipid-sterol or sphingolipid-sphingolipid interactions (sur2), respectively. Although lipid extracts from control yeast cells possess an inherent self-organization potential, resulting in liquid-disordered $\left(\mathrm{L}_{\mathrm{d}}\right) /$ liquid-ordered $\left(\mathrm{L}_{\mathrm{o}}\right)$ phase coexistence in giant unilamellar vesicles at lowered temperature, lipid extracts from the elo 3 and sur 2 mutant cells failed to phase separate (Klose et al. 2010). Surprisingly, these seemingly small changes in lipid structure in the yeast mutants have dramatic effects on the thermodynamics of yeast membrane organization. 
Membrane Organization and Lipid Rafts

Our working hypothesis is that the increase in raft lipids, ergosterol, and sphingolipids promotes a raft coalescence process induced by clustering of raft components (e.g., by lectins) that could lead to selective raft protein and lipid segregation in TGN membranes (Fig. 2). The immiscibility of the two liquid phases in the membrane bilayer introduces an energetic penalty that promotes membrane bending because of increased thickness and order of the raft domain compared to the more disordered vicinity (Schuck and Simons 2004; Klemm et al.
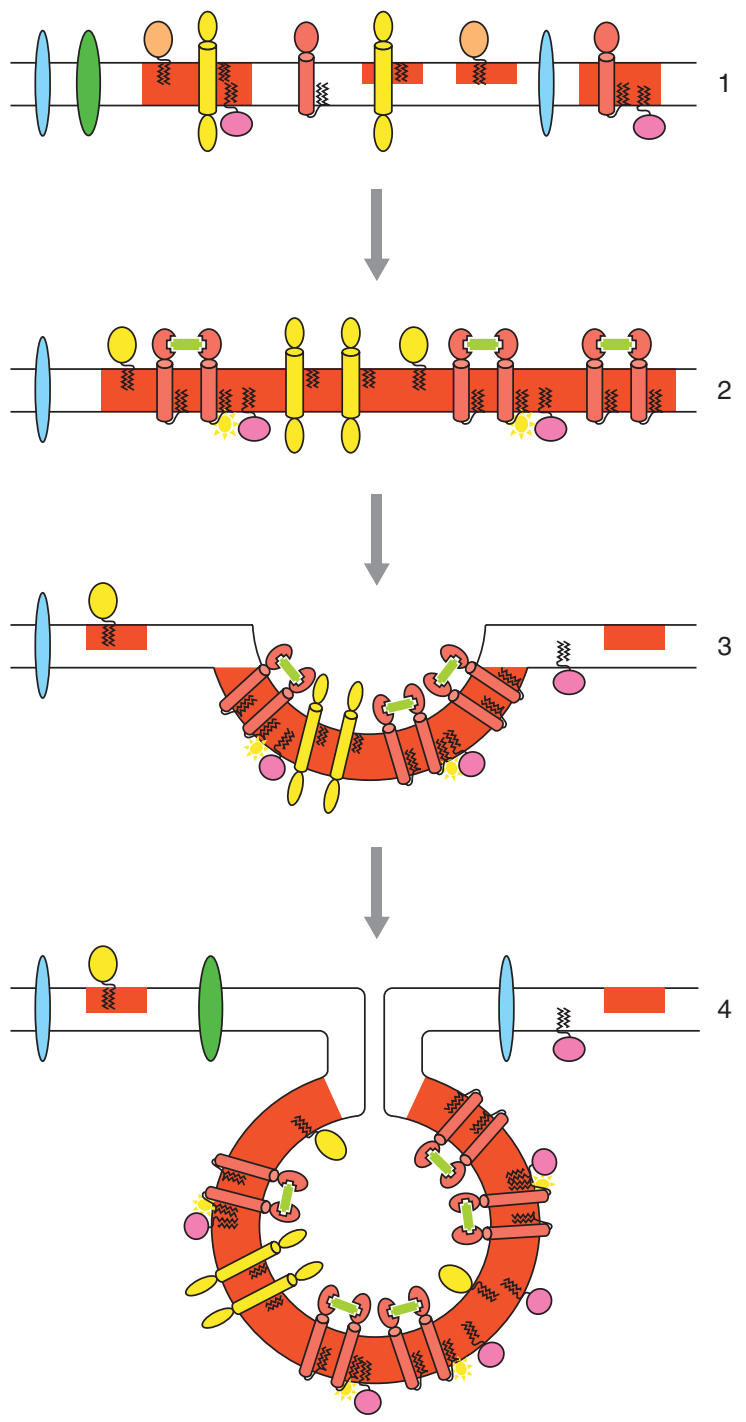

Figure 2. Raft clustering and domain-induced budding. Before clustering, proteins associate with rafts (red) to various extents (1). Clustering is induced, for example, by the binding of a dimerizing protein (green) to a transmembrane raft protein (2). The scaffolded raft-associated proteins coalesce into a raft cluster. Growth of the clustered raft domain beyond a critical size induces budding (3). Finally, a transport container consisting of raft components pinches off from the parent membrane by fission at the domain boundaries. Additional protein machinery will facilitate and regulate the budding process (4). 
2009). This bulk sorting of proteins and lipids is fine-tuned by specific sorting, aided by accessory proteins that bind to raft cargo, such as the Astlp protein that facilitates the delivery of Pmalp, the proton ATPase to the cell surface (Bagnat et al. 2001). Protein machinery involved in bending and release would also be required to bud the membrane domain into a transport vesicle, leading to regulated protein and lipid sorting at the exit from the TGN.

The apical membrane of epithelial cells has in two studies been shown to behave like a large raft membrane. Measuring long-range diffusion of several membrane proteins by FRAP in the apical membrane of MDCK cells as compared with the same protein in the PM of a fibroblast, the conclusion was that the apical membrane behaved as a percolating (continuous) phase at $25^{\circ} \mathrm{C}$, in which raft proteins freely diffused whereas nonraft proteins were dispersed into isolated domains (Meder et al. 2006). Also, the apical brush border membrane of small intestinal cells was described as behaving as a large super-raft domain stabilized by galectin- 4 and another lectin, intelectin (Danielsen and Hansen 2008).

These findings fit well with the data obtained in the lipidomic study of the polarized MDCK cell. The changes that accompanied cell polarization were what would be expected when an apical membrane is introduced into the cell during polarization (Sampaio et al. 2010). The remodeling of the lipidome conforms to the creation of a robust and impermeable barrier, composed of coalescing rafts. Complex glycosphingolipids like the Forssman pentasaccharide glycolipid could, together with cholesterol, generate a hydrogen-bonded network in the outer leaflet of the apical membrane that helps to protect the cell against the harsh external environment. At the same time, the proposed continuous recycling of galectin-9 and the Forssman lipid could serve the role of creating foci for raft domain coalescence in the TGN to facilitate apical transport carrier formation.

Future studies are needed to analyze how the lectin could function as the postulated nucleation device for generating the apical carrier and to identify the other proteins that participate in the process.

\section{THE DYNAMIC ORGANIZATION OF THE PLASMA MEMBRANE}

An enormous challenge to the field was to develop methodology to study the dynamic organization of cell membranes. The concept of sphingolipid-sterol-protein rafts that were smaller than the resolution of the optical microscope stimulated the search for novel methodologies. Recent studies using different imaging and spectroscopic methods have revealed interesting glimpses of how the lipids and proteins behave in the crowded bilayer (Sezgin and Schwille 2011). These studies have confirmed the existence of cholesteroldependent nanoscale assemblies of sphingolipids and GPI-anchored proteins in the plasma membrane of living cells. Also, atomic force measurements have been employed to show that GPI-anchored proteins reside in nanoscale rafts that are stiffer than the surrounding membrane (Roduit et al. 2008). The lifetime of the nanoscale assemblies seems to vary with the method used to do the measurements, as so does the spatial scale of the assemblies. Obviously, the state of these assemblies is easily influenced by the methods used to observe them. Although Kusumi et al. (2004) concluded from their single molecule imaging studies that the lifetime of the nanoscopic rafts is in the millisecond range, Brameshuber et al. (2010) observed with their special photobleaching protocol long-lived nanoplatforms that remained together for seconds.

There are different views to explain the existence of nanoscale rafts. Recent studies suggest that the composition of plasma membranes is tuned close to a critical miscibility point (Honerkamp-Smith et al. 2009). Critical points are defined in simple model membranes by special compositions and temperatures in the phase diagrams in which the coexisting $\mathrm{L}_{\mathrm{o}} / \mathrm{L}_{\mathrm{d}}$ phases approach identity and exist as interconverting fluctuations (Veatch et al. 2007). Critical behavior was observed in giant plasma membrane vesicles (plasma membrane 
preparations cooled to phase separate into two liquid phases following membrane blebbing from cells by chemical treatment) (Veatch et al. 2008). This astonishing finding implies that the large compositional fluctuations observed at room temperature could be equated with nanoscale rafts at physiological temperature. These results raise the intriguing possibility that the composition of plasma membranes is tuned to reside close to a critical point, facilitating membrane subcompartmentalization at little energetic cost.

Another interpretation is that the nanoscale rafts in cell membranes are analogous to microemulsions, in which fluctuations arise like in a ternary fluid mixture that contains an interfacially active agent (Hancock 2006; Brewster et al. 2009; Schäfer and Marrink 2010). Brewster and Safran (2010) have suggested that lipids that have one fully saturated chain and one partially unsaturated chain could function as a surface-active component, a hybrid lipid or a linactant. The linactants would lower the line tension between domains by occupying the interface, having the saturated anchor preferring the raft and the unsaturated fatty acid facing the less ordered lipid environment. In this way, finite-sized assemblies, stabilized by these hybrid lipids, could form as equilibrium structures (Brewster et al. 2009). Perhaps also proteins could act as linactants. Several protein structures would be ideally suited for this purpose. For instance, proteins that have both a GPI anchor and a trans-membrane domain have been identified, in which the GPI anchor could be raft-associated with the transmembrane domain facing the nonraft bilayer (Kupzig et al. 2003). Another protein is the influenza virus M2 protein, which has been postulated to occupy the perimeter of the raft domain, formed when the virus buds out of the plasma membrane (Schroeder et al. 2005; Rossman et al. 2010). Also, N-Ras has been proposed to act as a linactant in the cytosolic leaflet of a raft (Weise et al. 2009).

Another issue that will be important for understanding the dynamics of plasma membrane organization is the influence of the underlying cytocortex and the actin cytoskeleton (Viola and Gupta 2007; Andrews et al. 2008; Chichili and Rodgers 2009). High spatial and temporal resolution FRET microscopy has revealed a nonrandom distribution of nanoclusters of GPI-anchored proteins, dependent on cholesterol and actin (Goswami et al. 2008). The authors postulated that these clusters are nucleated by dynamic actin filaments using myosin-like motors. The same group also analyzed the nanoscale organization of Hedgehog, a well-studied signaling protein (Vyas et al. 2008). Hedgehog is anchored to the membrane by a cholesterol moiety and by palmitoylation. The FRET studies revealed that Hedgehog forms nanoscale oligomers that could be concentrated into visible clusters, capable of signaling. The lipid modifications were found to be important for the nanoscale organization. However, the role of actin in this process was not yet studied.

Kusumi et al. have pioneered a picket-fence model controlling lateral diffusion of membrane proteins and lipids (Ritchie et al. 2003). In this model, actin filaments apposed to the cytosolic side of the membrane would form hurdles impeding diffusion. A further suggestion is that trans-membrane proteins become transiently anchored to the actin filaments, acting as a row of pickets slowing the diffusion of other proteins and even of lipids. The picket lines could be observed by single molecule imaging at 25 - $\mu \mathrm{sec}$ time resolution, showing that proteins and lipids are undergoing shortterm confined diffusion before they "hop" over the barrier through a "hole" in the fence every 1-100 msec (Fujiwara et al. 2002; Murase et al. 2004; Morone et al. 2006). Other investigators using different methods failed to observe the hop diffusion of lipids (Sahl et al. 2010).

Recent studies have thus unveiled a bewildering plethora of behaviors that characterize the nanoscale organization or raft proteins and lipids. Clearly, different methods emphasize different characteristics of these dynamic structures that can only be reconciled by further work (for a discussion, see Brameshuber et al. 2010). 


\section{FUNCTIONALIZATION OF NANOSCALE RAFTS}

In living cells, raft assemblies can be stabilized by specific oligomerization of raft proteins or lipids with little energy input (Figs. 2 and 3). In this way, larger and more stable rafts are generated containing predominantly proteins that are brought into a specific raft domain by ligation and/or scaffolding. Raft affinity can be further enhanced by oligomerization (Dietrich et al. 2001; Sengupta et al. 2008; Levental et al. $2010 \mathrm{~b})$. The merger of specific nanoscale rafts into larger and more stable platforms represents the functionalization of specific rafts in membrane trafficking both in the biosynthetic and the endocytic pathways as well as in signal transduction and other raft-associated processes (Fig. 3) (Simons and Toomre 2000; Hancock 2006; Lingwood and Simons 2010; Simons and Gerl 2010).

The merger of rafts can also be induced experimentally by artificial means. Early studies showed that large raft domains could be induced by cross-linking raft components with antibodies (Fig. 3). The size of the resulting raft domain was determined by the extent of cross-linking. These studies led to the erroneous conclusion that raft markers such as GPIanchored proteins or lipids such as GM1 should be enriched in rafts. However, considering the widely varying spatial scale of raft domains between cross-linked rafts compared to fluctuating nanoscale assemblies in living cells, it becomes obvious that the inclusion of so-called "raft markers" is likely to be dependent on the state of the "rafts" being studied. In the fluctuating nanoscale rafts, the likelihood of specific raft proteins being together depends on their interactions with each other and with specific raft lipids. Most raft proteins will reside in individual spatially distinct nanoscopic rafts. Additionally, it is important to note that raft size and composition will depend on the cell membrane environment. For instance, resting state rafts in the apical membrane of an epithelial cell (Meder et al. 2006) will be different from those in the plasma membrane of a fibroblast or an immunocyte. Functionalization of resting state rafts will lead to yet another organization, depending on the how the merger of specific nanoscale rafts is mediated (Simons and Toomre 2000; Hancock 2006).

One open issue is the mechanism of coupling between the two leaflets in rafts (Kiessling et al. 2006; Collins and Keller 2008). It has been observed that cytosolic proteins, lipidated with two saturated fatty acyls localize underneath coalescing rafts (Harder et al. 1998; Gri et al. 2004). However, the mechanism of this coupling of the exoplasmic leaflet with the cytosolic leaflet remains unknown. One possibility is that the long fatty acids present in many sphingolipids could intercalate into the inner leaflet. An ordered outer leaflet would bring order into the underlying inner leaflet lipid species. Also how the lipid composition of the cytosolic leaflet in rafts is composed and regulated is yet to be explained.

\section{PHASE SEPARATION IN PLASMA MEMBRANES}

In model membranes containing simple lipid mixtures, microscopic phase separation can easily be induced by adjusting the composition and temperature of lipid bilayers (Heberle and Feigenson 2011). Such phase separation was not thought to be possible in complex mixtures, like those in cell membranes. However, recent studies surprisingly show that compositionally complex plasma membranes can also be induced to phase segregate into two fluid domains. Baumgart et al. (2007) showed that cells treated with paraformaldehyde and dithiothreitol produced membrane blebs that could be isolated as giant plasma membrane vesicles. When chilled below room temperature, these membranes phase separated into $\mathrm{L}_{\mathrm{o}}$-like and $\mathrm{L}_{\mathrm{d}}$-like phases. The temperature and cholesterol dependence of this phase separation resembled that of simple model systems (Levental et al. 2009). Other studies of plasma membranes blown up into giant spheres using a swelling procedure that separated the membranes from the influence of cytoskeletal and membrane trafficking processes showed cholesterol-dependent coalescence into micrometer-scale phases on 
Resting state

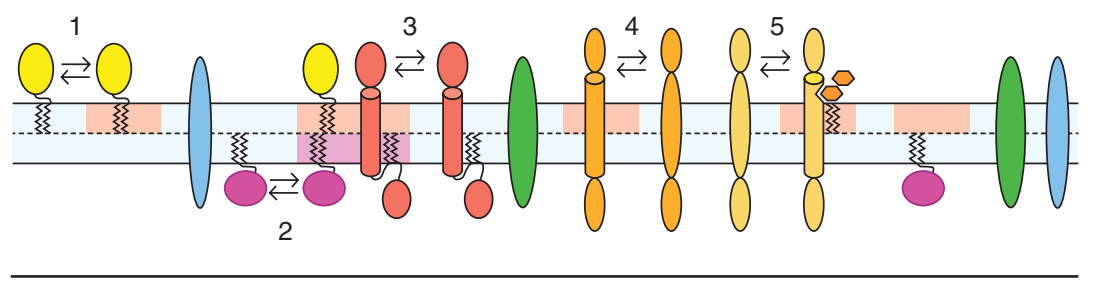

Activated, clustered rafts

6

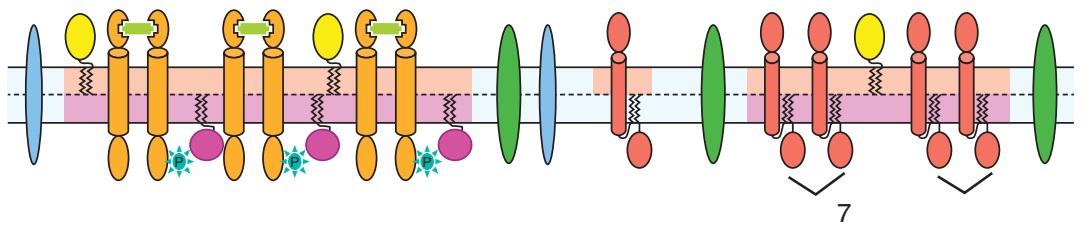

Large raft cluster

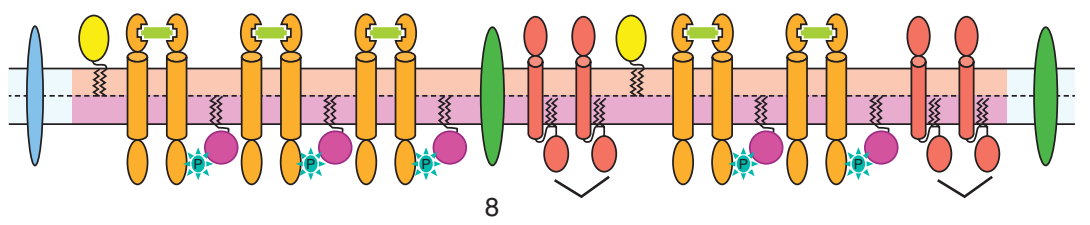

8

Nonraft domain

Sphingolipid-cholesterol raft domain

in exoplasmic leaflet

Ordered lipid domain in cytoplasmic

leaflet

GPI-anchored protein

Doubly acylated protein

$\left\{\begin{array}{l}\text { Acylated transmembrane raft } \\ \text { protein }\end{array}\right.$

srotein

$\checkmark$ Cytoplasmic scaffolding protein
Transmembrane protein that changes its conformation upon partitioning into rafts

$\beta$ Transmembrane raft protein that binds glycosphingolipid

Two different nonraft transmembrane proteins

Phosphorylated transmembrane raft
protein

Figure 3. The tunable states of rafts. Resting-state rafts are dynamic, nanoscopic assemblies of raft lipids and proteins that are metastable (i.e., persist for a certain time $[t o p]$ ). The coupling between the outer and the inner leaflet is not well understood. Most raft proteins are either solely lipid-anchored (GPI-anchored in the exoplasmic [1] or doubly acylated in the cytoplasmic leaflet [2]), or they contain acyl chains in addition to their TMD (3). A fourth group could undergo a conformational change when partitioning into rafts (4) or following binding to glycosphingolipids (5). Following oligomerization of raft proteins by multivalent ligands (6) or cytoplasmic scaffolds (7), the small raft domains coalesce and become more stable. They may now contain more than one family of raft proteins. These small raft clusters would still have a size below the limits of light microscopic resolution, but could already function as signaling platforms. Large raft clusters are probably only assembled when protein modifications like phosphorylation increase the number of protein-protein interactions, leading to the coalescence of small clusters into larger domains on the scale of several hundred nanometers (8). 
clustering of the ganglioside GM1 by cholera toxin at $37^{\circ} \mathrm{C}$ (Lingwood et al. 2008). Most astonishing and different from the behavior in the PM blebs, trans-membrane PM proteins that have been predicted to associate with rafts by detergent resistance and other assays, partitioned into the phase containing the ganglioside GM1 (cross-linked by the cholera toxin). The selective lateral reorganization of PM proteins and lipids in the phase separated PM spheres correlated with their predicted affinity for raft domains. In contrast, in the PM blebs formed after treatment with paraformaldehyde and dithiothreitol, raft trans-membrane proteins were excluded from the $\mathrm{L}_{\mathrm{O}}$-like phase (Sengupta et al. 2008; Levental et al. 2010b).

Another remarkable analogy between the behavior of simple model systems and the plasma membranes that are composed of hundreds of lipids and proteins is that cholera toxin-induced phase separation has also been observed in ternary lipid mixtures of unsaturated PC, sphingomyelin, and cholesterol (containing the ganglioside GM1) (Hammond et al. 2005). Importantly, this induction is only observed when the lipid mixture in the model system is positioned compositionally close to a phase boundary, implying that the protein and lipid composition of the plasma membrane is also positioned close to a phase boundary.

Altogether, these and other studies (Ayuyan and Cohen 2008) show that plasma membrane composition is poised for selective coalescence at physiological temperature. They highlight the inherent capability of the PM to phase separate while stressing that in the living cell this capacity is strictly controlled by the lipid and protein composition of the membrane as well as by the fact that cell membranes are not at equilibrium, being continuously perturbed by exchange events and membrane trafficking. It is also important to point out that in all phaseseparated PMs, the actin cytoskeleton has been removed, probably resulting from PIP2 hydrolysis. This facilitates the formation of large micrometer domains unimpeded by actin barriers.

These exciting findings also emphasize that the coalescence of a micrometer raft phase can only be brought about by the merger of small (nanoscale) rafts composed of lipids and proteins already present in the plasma membrane. Obviously, the lipid and protein composition has to be such that the merger into micrometer raft domains becomes energetically possible.

\section{LIPID-PROTEIN INTERACTIONS IN RAFTS}

The analogy with phase-separating simple model systems of ternary lipids and sphingolipid- and sterol-containing cell membranes breaks down when it comes to the partitioning behavior of raft trans-membrane proteins. These are usually excluded from the $\mathrm{L}_{\mathrm{o}}$ phase in reconstituted proteoliposomes (Sengupta et al. 2008; Levental et al. 2010b). Cell membranes are crowded with proteins that have different affinities for raft domains. How is this affinity controlled? What makes a transmembrane protein raftophilic? Previous studies on this issue were based on detergent-resistance. The indirect and controversial nature of these experiments limited their applicability in assigning raft affinity. With the advent of phaseseparated PMs, the analysis of raftophilicity is becoming more straightforward. By fluorescently tagging trans-membrane proteins and expressing them in cells, their partitioning in phase-separated PMs can be measured quantitatively by confocal microscopy. In this way, Levental et al. (2010b) showed that palmitoylation plays an important role in regulating raft affinity. The reason for the exclusion of the raft proteins from the raft phase in the chemically induced blebs was the use of dithiothreitol, which led to depalmitoylation by thioester reduction. Because cysteine palmitoylation is the only posttranslational lipid modification of proteins that has been shown to be reversible regulated, these data suggest a role for palmitoylation as dynamic raft targeting mechanism for trans-membrane proteins (Resh 2006). However, it is important to point out that palmitoylation is definitely not sufficient for raft association. There are many palmitoylated proteins that are not raft-associated, including the generally used nonraft marker, the transferrin receptor. Both TMD length and amino acid 
sequence will be involved in defining raftophilicity (Fig. 4A) (Scheiffele et al. 1997; Barman and Nayak 2000; Engel et al. 2010).

Another lipid known to promote raft association is the GPI anchor. There are different chemical types of GPI anchors not all of which necessarily raftophilic, though the different anchors have not yet been analyzed for raft affinity (Ferguson et al. 2009). Levental et al. used both depalmitoylation by dithiothreitol treatment and removal of GPI anchors by a GPI-specific phospholipase to determine the percentage of PM proteins that were partitioning into the raft phase of phase segregated PMs (Levental et al. 2010b). About $65 \%$ of the PM proteins were in the nonraft phase, whereas $12 \%$ of the integral proteins required palmitoylation for raft phase inclusion. About 11\% were GPI-anchored in the raft phase and another $11 \%$ was sensitive to neither treatment; therefore the mechanism of raft association remained unassigned (Fig. 4B). This group of proteins could be bound to raft lipids such as cholesterol or sphingolipids (Contreras et al. 2011). Thus, there will be several means for associating proteins with sphingolipids-sterol rafts. Elucidating how proteins become lubricated to achieve raft affinity, and how this raft affinity could regulate protein function, will be an issue for future research. For example, binding to a specific raft lipid has recently been shown to allosterically change the conformation of the human epidermal growth factor receptor (Coskun et al. 2011). Thus, the functional association of proteins with rafts would not only compartmentalize the membrane-bound process but also induce conformational changes that modulate protein function.

\section{CONCLUDING REMARKS}

The increasing insights into the dynamics of cell membrane organization have highlighted the need for the regulation of the compositional diversity of membrane lipid. The pioneering work of Brown and Goldstein showed that the transcription of genes controlling cholesterol level is directly regulated by the concentration of cholesterol in the ER of mammals (Brown and Goldstein 2009). Sensors in the elaborate SREBP pathway lead to tight control of cholesterol homeostasis. Similarly, glycerolipids have been shown to be regulated by multiple feedback mechanisms that link synthesis and degradation of these lipids to their cellular levels
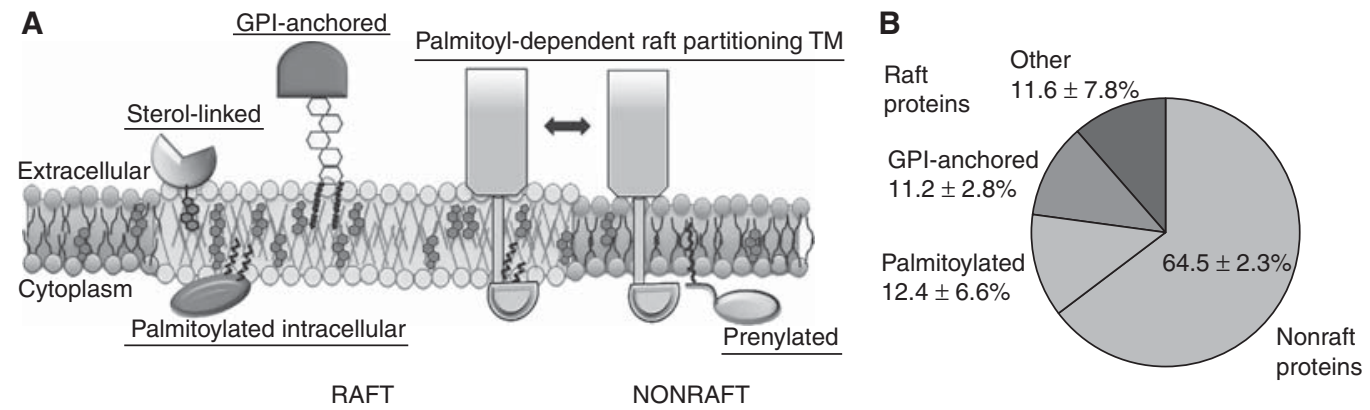

Figure 4. Lipid modifications of proteins as determinants of raft association. (A) Examples of lipid modification of proteins. Various lipid anchors play important roles in protein trafficking, membrane partitioning and proper function, likely mediated by their affinity for lipid rafts. The general paradigm is that anchoring by saturated fatty acids and sterols targets proteins to the more tightly packed environment of lipid rafts, whereas unsaturated and branched hydrocarbon chains tend to favor the less restrictive nonraft membranes. Palmitoylation of proteins can regulate raft partitioning. ( $A$, Adapted from Levental et al. 2010a; reprinted with permission from the American Chemical Society (C) 2010.) (B) Quantification of raft protein abundance following removal of palmitoylated TM proteins by DTT or GPI-anchored proteins by GPI-specific phospholipase in GPMVs (average + SD from three independent experiments). ( $B$, Adapted from Levental et al. 2010b; reprinted with permission from The National Academy of Sciences (C) 2010.) 
(Nohturfft and Zhang 2009). Recent studies are also giving insights into how the sphingolipids are regulated (Vacaru et al. 2009; Breslow and Weissman 2010). There is also an increasing body of evidence that sterol and sphingolipid metabolism are closely coordinated (Hannich et al. 2011). Thus, there is a need for sensors that can measure levels of different lipids and provide feedback into the control systems that regulate lipid homeostasis. Interestingly, recent findings have showed that a bacterial protein can work as a thermometer and detect changes in environmental temperature by physically measuring membrane thickness (Cybulski et al. 2010).

Obviously, if the composition of the plasma membranes of fibroblasts and other cells has been positioned close to a phase boundary or to a critical immiscibility point, then the protein and lipid composition needs to be strictly fine-tuned. Nutritional research has stressed the importance of the right distribution of fatty acids in lipid molecules. For instance, the levels of fatty acids with omega-3 unsaturation have been suggested to be important for health (Riediger et al. 2009). Until now, no lipidomic studies have been performed analyzing the full diversity of lipidomes with respect to the influence of fatty acid content of the diet. Obviously, the diet can in the long run lead to imbalances and modulate the fine-tuning of lipid levels such that disease is caused, for instance myocardial infection through atherosclerosis (Puska 2009). Here is an interesting new area of research that will profit from the enormous advances in lipid analyses by mass spectrometry (Schwudke et al. 2011). Inbuilt into the functions of the compositional diversity of all membranes, there must also be feedback mechanisms introducing robustness so that the structure and function of cellular membranes is maintained despite varying lipid intake. These are areas of research that can now be explored by multidisciplinary approaches.

What is emerging from recent cell membrane research is a fascinating two-dimensional liquid equipped with remarkable properties. Most intriguing is the concept of collectives of lipids and proteins that work together to make cell membranes such incredible matrices for supporting and facilitating cellular function.

\section{ACKNOWLEDGMENTS}

We thank Hermann-Josef Kaiser and Ilya Levental for reading the paper and the Simons lab for continuous critical input. We also thank Doris Meder, especially for drawing Figure 3. This work was supported by DFG "Schwerpunktprogramm1175” Grant no. SI459/2-1, DFG "Transregio 83" Grant no. TRR83 TP02, BMBF "ForMaT" Grant no. 03FO1212, ESF "LIPIDPROD” Grant no. SI459/3-1, and the Klaus Tschira Foundation.

\section{REFERENCES}

Andrews NL, Lidke KA, Pfeiffer JR, Burns AR, Wilson BS, Oliver JM, Lidke DS. 2008. Actin restricts Fce RI diffusion and facilitates antigen-induced receptor immobilization. Nat Cell Biol 10: 955-963.

Ayuyan AG, Cohen FS. 2008. Raft composition at physiological temperature and $\mathrm{pH}$ in the absence of detergents. Biophys J 94: 2654-2666.

Bagnat M, Chang A, Simons K. 2001. Plasma membrane proton ATPase Pmalp requires raft association for surface delivery in yeast. Mol Biol Cell 12: 4129-4138.

Bagnat M, Keraenen S, Shevchenko A, Shevchenko A, Simons K. 2000. Lipid rafts function in biosynthetic delivery of proteins to the cell surface in yeast. Proc Natl Acad Sci 97: 3254-3259.

Barman S, Nayak DP. 2000. Analysis of the transmembrane domain of influenza virus neuraminidase, a type II transmembrane glycoprotein, for apical sorting and raft association. J Virol 74: 6538-6545.

Baumgart T, Hammond AT, Sengupta P, Hess ST, Holowka DA, Baird BA, Webb WW. 2007. Large-scale fluid/fluid phase separation of proteins and lipids in giant plasma membrane vesicles. Proc Natl Acad Sci 104: 3165-3170.

Brameshuber M, Weghuber J, Ruprecht V, Gombos I, Horvát I, Vigh Ls, Eckerstorfer P, Kiss E, Stockinger H, Schütz GJ. 2010. Imaging of mobile long-lived nanoplatforms in the live cell plasma membrane. J Biol Chem 285: 41765-41771.

Breslow DK, Weissman JS. 2010. Membranes in balance: Mechanisms of sphingolipid homeostasis. Mol Cell 40: 267-279.

Bretscher MS, Munro S. 1993. Cholesterol and the Golgi apparatus. Science 261: 1280-1281.

Brewster R, Safran SA. 2010. Line active hybrid lipids determine domain size in phase separation of saturated and unsaturated lipids. Biophys J 98: L21-L23.

Brewster R, Pincus PA, Safran SA. 2009. Hybrid lipids as a biological surface-active component. Biophys J 97: 1087-1094. 
Brown DA, Rose JK. 1992. Sorting of GPI-anchored proteins to glycolipid-enriched membrane subdomains during transport to the apical cell surface. Cell 68: $533-544$.

Brown MS, Goldstein JL. 2009. Cholesterol feedback: From Schoenheimer's bottle to Scap's MELADL. J Lipid Res 50: S15-S27.

Brugger B, Sandhoff R, Wegehingel S, Gorgas K, Malsam J, Helms JB, Lehmann WD, Nickel W, Wieland FT. 2000. Evidence for segregation of sphingomyelin and cholesterol during formation of COPI-coated vesicles. J Cell Biol 151: 507-518.

Chichili G, Rodgers W. 2009. Cytoskeleton-membrane interactions in membrane raft structure. Cell Mol Life Sci 66: 2319-2328.

Collins MD, Keller SL. 2008. Tuning lipid mixtures to induce or suppress domain formation across leaflets of unsupported asymmetric bilayers. Proc Natl Acad Sci 105: $124-128$.

Contreras F-X, Ernst AM, Wieland F, Brügger B. 2011. Specificity of intramembrane protein-lipid interactions. Cold Spring Harb Perspect Biol 3: a004705.

Coskun Ü, Simons K. 2010. Membrane rafting: From apical sorting to phase segregation. FEBS Lett 584: 1685-1693.

Coskun U, Grzybek M, Dreschsel D, Simons K. 2011. Allosteric regulation of human EGF receptor by lipids. Proc Natl Acad Sci (in press).

Cybulski LE, Martín M, Mansilla MC, Fernández A, de Mendoza D. 2010. Membrane thickness cue for cold sensing in a bacterium. Curr Biol 20: 1539-1544.

Danielsen E, Hansen G. 2008. Lipid raft organization and function in the small intestinal brush border. J Physiol Biochem 64: 377-382.

Delacour D, Gouyer V, Zanetta J-P, Drobecq H, Leteurtre E, Grard G, Moreau-Hannedouche O, Maes E, Pons A, André S, et al. 2005. Galectin-4 and sulfatides in apical membrane trafficking in enterocyte-like cells. J Cell Biol 169: 491-501.

Dietrich C, Volovyk ZN, Levi M, Thompson NL, Jacobson K. 2001. Partitioning of Thy-1, GM1, and cross-linked phospholipid analogs into lipid rafts reconstituted in supported model membrane monolayers. Proc Natl Acad Sci 98: 10642-10647.

Engel S, Scolari S, Thaa B, Krebs N, Korte T, Herrmann A, Veit M. 2010. FLIM-FRET and FRAP reveal association of influenza virus haemagglutinin with membrane rafts. Biochem J 425: 567-573.

Engelman DM. 2005. Membranes are more mosaic than fluid. Nature 438: 578-580.

Ferguson M, Kinoshita T, Hart G. 2009. Glycosylphosphatidylinositol anchors. In Essentials of glycobiology, 2nd ed. (ed. Varki A, et al.). Cold Spring Harbor Laboratory Press, Cold Spring Harbor, NY.

Fiedler K, Kobayashi T, Kurzchalia TV, Simons K. 1993. Glycosphingolipid-enriched, detergent-insoluble complexes in protein sorting in epithelial cells. Biochemistry 32: 6365-6373.

Friedrichs J, Torkko JM, Helenius J, Teraevaeinen TP, Fuellekrug J, Muller DJ, Simons K, Manninen A. 2007. Contributions of Galectin-3 and - 9 to epithelial cell adhesion analyzed by single cell force spectroscopy. J Biol Chem 282: 29375-29383.
Frolov VA, Shnyrova AV, Zimmerberg J. 2011. Lipid polymorphisms and membrane shape. Cold Spring Harb Perspect Biol doi:10.1101/cshperspect.a004747.

Fujiwara T, Ritchie K, Murakoshi H, Jacobson K, Kusumi A. 2002. Phospholipids undergo hop diffusion in compartmentalized cell membrane. J Cell Biol 157: 1071-1082.

Futerman AH, Hannun YA. 2004. The complex life of simple sphingolipids. $E M B O$ 5: 777-782.

Gorter E, Grendel F. 1925. On bimolecular layers of lipoids on the chromocytes of the blood. J Exp Med 41: 439-443.

Goswami D, Gowrishankar K, Bilgrami S, Ghosh S, Raghupathy R, Chadda R, Vishwakarma R, Rao M, Mayor S. 2008. Nanoclusters of GPI-anchored proteins are formed by cortical actin-driven activity. Cell 135: 1085-1097.

Gri G, Molon B, Manes S, Pozzan T, Viola A. 2004. The inner side of T cell lipid rafts. Immunol Lett 94: 247-252.

Gurunathan S, David D, Gerst JE. 2002. Dynamin and clathrin are required for the biogenesis of a distinct class of secretory vesicles in yeast. EMBO J 21: 602-614.

Hammond AT, Heberle FA, Baumgart T, Holowka D, Baird B, Feigenson GW. 2005. Crosslinking a lipid raft component triggers liquid ordered-liquid disordered phase separation in model plasma membranes. Proc Natl Acad Sci 102: 6320-6325.

Hancock JF. 2006. Lipid rafts: Contentious only from simplistic standpoints. Nat Rev Mol Cell Biol 7: 456-462.

Hannich JT, Umebayashi K, Riezman H. 2011. Distribution and functions of sterols and sphingolipids. Cold Spring Harb Perspect Biol doi:10.1101/cshperspect.a004762.

Harder T, Scheiffele P, Verkade P, Simons K. 1998. Lipid domain structure of the plasma membrane revealed by patching of membrane components. J Cell Biol 141: 929-942.

Harsay E, Bretscher A. 1995. Parallel secretory pathways to the cell surface in yeast. J Cell Biol 131: 297-310.

Heberle FA, Feigensen GW. 2011. Phase separation in lipid membranes. Cold Spring Harb Perspect Biol 3: a004630.

Honerkamp-Smith AR, Veatch SL, Keller SL. 2009. An introduction to critical points for biophysicists; observations of compositional heterogeneity in lipid membranes. Biochim Biophys Acta 1788: 53-63.

Jacobson K, Mouritsen OG, Anderson RGW. 2007. Lipid rafts: At a crossroad between cell biology and physics. Nat Cell Biol 9: 7-14.

Kawai K, Fujita M, Nakao M. 1974. Lipid components of two different regions of an intestinal epithelial cell membrane of mouse. Biochim Biophys Acta 369: $222-233$.

Keller P, Simons K. 1998. Cholesterol is required for surface transport of influenza virus hemagglutinin. J Cell Biol 140: $1357-1367$.

Kiessling V, Crane JM, Tamm LK. 2006. Transbilayer effects of raft-like lipid domains in asymmetric planar bilayers measured by single molecule tracking. Biophys J 91: 3313-3326.

Klemm RW, Ejsing CS, Surma MA, Kaiser HJ, Gerl MJ, Sampaio JL, de Robillard Q, Ferguson C, Proszynski TJ, Shevchenko A, et al. 2009. Segregation of sphingolipids and sterols during formation of secretory vesicles at the trans-Golgi network. J Cell Biol 185: 601-612. 
Klose C, Ejsing CS, Garcia-Saez AJ, Kaiser HJ, Sampaio JL, Surma MA, Shevchenko A, Schwille P, Simons K. 2010. Yeast lipids can phase-separate into micrometerscale membrane domains. J Biol Chem 285: $30224-$ 30232.

Kupzig S, Korolchuk V, Rollason R, Sugden A, Wilde A, Banting G. 2003. Bst-2/HM1.24 is a raft-associated apical membrane protein with an unusual topology. Traffic 4: 694-709.

Kusumi A, Koyama-Honda I, Suzuki K. 2004. Molecular dynamics and interactions for creation of stimulationinduced stabilized rafts from small unstable steady-state rafts. Traffic 5: 213-230.

Levental I, Grzybek M, Simons K. 2010a. Greasing their way: Lipid modifications determine protein association with membrane rafts. Biochemistry 49: 6305-6316.

Levental I, Byfield FJ, Chowdhury P, Gai F, Baumgart T, Janmey PA. 2009. Cholesterol-dependent phase separation in cell-derived giant plasma-membrane vesicles. Biochem J 424: 163-167.

Levental I, Lingwood D, Grzybek M, Coskun U, Simons K. 2010b. Palmitoylation regulates raft affinity for the majority of integral raft proteins. Proc Natl Acad Sci 107: 22050-22054.

Lingwood D, Simons K. 2010. Lipid rafts as a membraneorganizing principle. Science 327: 46-50.

Lingwood D, Ries J, Schwille P, Simons K. 2008. Plasma membranes are poised for activation of raft phase coalescence at physiological temperature. Proc Natl Acad Sci 105: $10005-10010$.

Lundbæk JA, Andersen OS, Werge T, Nielsen C. 2003. Cholesterol-induced protein sorting: An analysis of energetic feasibility. Biophys J 84: 2080-2089.

Mays RW, Siemers KA, Fritz BA, Lowe AW, van Meer G, Nelson WJ. 1995. Hierarchy of mechanisms involved in generating $\mathrm{Na} / \mathrm{K}$-ATPase polarity in MDCK epithelial cells. J Cell Biol 130: 1105-1115.

Meder D, Moreno MJ, Verkade P, Vaz WL, Simons K. 2006 Phase coexistence and connectivity in the apical membrane of polarized epithelial cells. Proc Natl Acad Sci 103: $329-334$

Mishra R, Grzybek M, Niki T, Hirashima M, Simons K. 2010. Galectin-9 trafficking regulates apical-basal polarity in Madin-Darby canine kidney epithelial cells. Proc Natl Acad Sci 107: 17633-17638.

Morone N, Fujiwara T, Murase K, Kasai RS, Ike H, Yuasa S, Usukura J, Kusumi A. 2006. Three-dimensional reconstruction of the membrane skeleton at the plasma membrane interface by electron tomography. J Cell Biol 174: $851-862$.

Mouritsen OG. 2005. Life-As a matter of fat. The emerging science of lipidomics, pp. 1-78. Springer-Verlag, Heidelberg.

Mouritsen O, Zuckermann M. 2004. What's so special about cholesterol? Lipids 39: 1101-1113.

Mouritsen OG. 2011. Model answers to lipid membrane questions. Cold Spring Harb Perspect Biol doi:10.1101/ cshperspect.a004622.

Murase K, Fujiwara T, Umemura Y, Suzuki K, Iino R, Yamashita H, Saito M, Murakoshi H, Ritchie K, Kusumi A. 2004. Ultrafine membrane compartments for molecular diffusion as revealed by single molecule techniques. Biophys J 86: 4075-4093.

Nohturfft A, Zhang SC. 2009. Coordination of lipid metabolism in membrane biogenesis. Annu Rev Cell Dev Biol 25: $539-566$.

Proszynski TJ, Klemm RW, Gravert M, Hsu PP, Gloor Y, Wagner J, Kozak K, Grabner H, Walzer K, Bagnat M, et al. 2005. A genome-wide visual screen reveals a role for sphingolipids and ergosterol in cell surface delivery in yeast. Proc Natl Acad Sci 102: 17981-17986.

Puska P. 2009. Fat and heart disease: Yes we can make a change-The case of North Karelia (Finland). Ann Nutr Metab 54: 33-38.

Resh MD. 2006. Palmitoylation of ligands, receptors, and intracellular signaling molecules. Sci STKE 2006: re14.

Riediger ND, Othman RA, Suh M, Moghadasian MH. 2009. A systemic review of the roles of $n-3$ fatty acids in health and disease. J Am Diet Assoc 109: 668-679.

Ritchie K, Iino R, Fujiwara T, Murase K, Kusumi A. 2003. The fence and picket structure of the plasma membrane of live cells as revealed by single molecule techniques. Mol Membr Biol 20: 13-18.

Rodriguez-Boulan E, Kreitzer G, Musch A. 2005. Organization of vesicular trafficking in epithelia. Nat Rev Mol Cell Biol 6: 233-247.

Roduit C, van der Goot FG, De Los Rios P, Yersin A, Steiner P, Dietler G, Catsicas S, Lafont F, Kasas S. 2008. Elastic membrane heterogeneity of living cells revealed by stiff nanoscale membrane domains. Biophys $J$ 94: $1521-1532$.

Rossman JS, Jing X, Leser GP, Lamb RA. 2010. Influenza virus M2 protein mediates ESCRT-independent membrane scission. Cell 142: 902-913.

Sahl SJ, Leutenegger M, Hilbert M, Hell SW, Eggeling C. 2010. Fast molecular tracking maps nanoscale dynamics of plasma membrane lipids. Proc Natl Acad Sci 107: 6829-6834.

Sampaio JL, Gerl MJ, Klose C, Ejsing CS, Beug H, Simons K, Shevchenko A. 2010. Membrane lipidome of an epithelial cell line. Proc Natl Acad Sci 108: 1903-1907.

Schäfer LV, Marrink SJ. 2010. Partitioning of lipids at domain boundaries in model membranes. Biophys $J$ 99: L91-L93.

Scheiffele P, Roth MG, Simons K. 1997. Interaction of influenza virus haemagglutinin with sphingolipid-cholesterol membrane domains via its transmembrane domain. EMBO J 16: 5501-5508.

Schroeder C, Heider H, Möncke-Buchner E, Lin T-I. 2005. The influenza virus ion channel and maturation cofactor M2 is a cholesterol-binding protein. Eur Biophys $J$ 34: $52-66$.

Schuck SSimons K. 2004. Polarized sorting in epithelial cells: Raft clustering and the biogenesis of the apical membrane. J Cell Sci 117: 5955-5964.

Schwudke D, Schuhmann K, Herzog R, Bornstein SR, Schevchenko A. 2011. Shotgun lipodomics on high resolution mass spectrometers. Cold Spring Harb Perspect Biol 3: a004614.

Seddon JM, Templer RH, Warrender NA, Huang Z, Cevc G, Marsh D. 1997. Phosphatidylcholine-fatty acid membranes: Effects of headgroup hydration on the phase 
behaviour and structural parameters of the gel and inverse hexagonal $\left(\mathrm{H}_{\mathrm{II}}\right)$ phases. Biochim Biophys Acta 1327: 131-147.

Sengupta P, Hammond A, Holowka D, Baird B. 2008 Structural determinants for partitioning of lipids and proteins between coexisting fluid phases in giant plasma membrane vesicles. Biochim Biophys Acta 1778: 20-32.

Sezgin E, Schwille P. 2011. Fluorescence techniques to study lipid dynamics. Cold Spring Harb Perspect Biol doi:10.1101/cshperspect.a009803.

Sharpe HJ, Stevens TJ, Munro S. 2010. A comprehensive comparison of transmembrane domains reveals organelle-specific properties. Cell 142: 158-169.

Shevchenko A, Simons K. 2010. Lipidomics: Coming to grips with lipid diversity. Nat Rev Mol Cell Biol 11: 593-598.

Simons K, Gerl MJ. 2010. Revitalizing membrane rafts: New tools and insights. Nat Rev Mol Cell Biol 11: 688-699.

Simons K, Ikonen E. 1997. Functional rafts in cell membranes. Nature 387: 569-572.

Simons K, Toomre D. 2000. Lipid rafts and signal transduction. Nat Rev Mol Cell Biol 1: 31-39.

Simons K, Van Meer G. 1988. Lipid sorting in epithelial cells. Biochemistry 27: 6197-6202.

Skibbens JE, Roth MG, Matlin KS. 1989. Differential extractability of influenza virus hemagglutinin during intracellular transport in polarized epithelial cells and nonpolar fibroblasts. J Cell Biol 108: 821-832.

Vacaru AM, Tafesse FG, Ternes P, Kondylis V, Hermansson M, Brouwers JFHM, Somerharju P, Rabouille C,
Holthuis JCM. 2009. Sphingomyelin synthase-related protein SMSr controls ceramide homeostasis in the ER. J Cell Biol 185: 1013-1027.

Vance J, Vance D, ed. 2008. Biochemistry of lipids, lipoproteins and membranes, pp. 1-39. Elsevier, Amesterdam.

van Meer G. 2011. Dynamic transbilayer lipid asymmetry. Cold Spring Harb Perspect Biol 3: a004671.

Veatch SL, Cicuta P, Sengupta P, Honerkamp-Smith A, Holowka D, Baird B. 2008. Critical fluctuations in plasma membrane vesicles. ACS Chem Biol 3: 287-293.

Veatch SL, Soubias O, Keller SL, Gawrisch K. 2007. Critical fluctuations in domain-forming lipid mixtures. Proc Natl Acad Sci 104: 17650-17655.

Viola A, Gupta N. 2007. Tether and trap: Regulation of membrane-raft dynamics by actin-binding proteins. Nat Rev Immunol 7: 889-896.

Vyas N, Goswami D, Manonmani A, Sharma P, Ranganath HA, VijayRaghavan K, Shashidhara LS, Sowdhamini R, Mayor S. 2008. Nanoscale organization of hedgehog is essential for long-range signaling. Cell 133: 1214-1227.

Weise K, Triola G, Brunsveld L, Waldmann H, Winter R. 2009. Influence of the lipidation motif on the partitioning and association of $\mathrm{N}$-Ras in model membrane subdomains. J Am Chem Soc 131: 1557-1564.

Wenk MR. 2010. Lipidomics: New tools and applications. Cell 143: 888-895.

Ye J, DeBose-Boyd RA. 2011. Regulation of cholesterol and fatty acid synthesis. Cold Spring Harb Perspect Biol 3: a004754. 


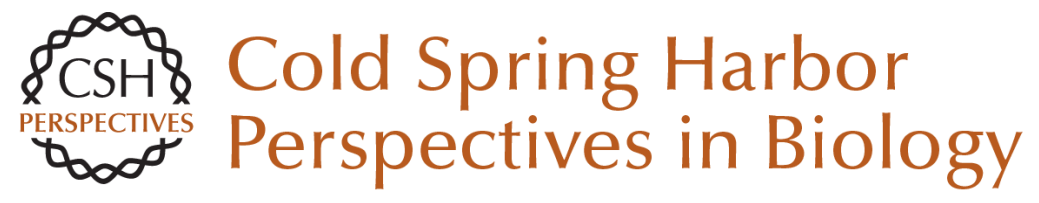

\section{Membrane Organization and Lipid Rafts}

Kai Simons and Julio L. Sampaio

Cold Spring Harb Perspect Biol 2011; doi: 10.1101/cshperspect.a004697 originally published online May 31, 2011

Subject Collection The Biology of Lipids

Role of Lipids in Virus Replication Maier Lorizate and Hans-Georg Kräusslich

Model Answers to Lipid Membrane Questions Ole G. Mouritsen

\section{Glycosphingolipid Functions} Clifford A. Lingwood

Regulation of Cholesterol and Fatty Acid Synthesis

Jin Ye and Russell A. DeBose-Boyd

\section{Lipid-Mediated Endocytosis}

Helge Ewers and Ari Helenius

\section{Fluorescence Techniques to Study Lipid \\ Dynamics}

Erdinc Sezgin and Petra Schwille

Lysosomal Lipid Storage Diseases

Heike Schulze and Konrad Sandhoff

\section{Distribution and Functions of Sterols and} Sphingolipids

J. Thomas Hannich, Kyohei Umebayashi and Howard Riezman
Membrane Organization and Lipid Rafts Kai Simons and Julio L. Sampaio

Shotgun Lipidomics on High Resolution Mass

Spectrometers

Dominik Schwudke, Kai Schuhmann, Ronny

Herzog, et al.

Glycosphingolipid Functions Clifford A. Lingwood

Phosphoinositides in Cell Architecture Annette Shewan, Dennis J. Eastburn and Keith Mostov

Synthesis and Biosynthetic Trafficking of Membrane Lipids Tomas Blom, Pentti Somerharju and Elina Ikonen

Lipid Polymorphisms and Membrane Shape Vadim A. Frolov, Anna V. Shnyrova and Joshua Zimmerberg

Specificity of Intramembrane Protein-Lipid Interactions

Francesc-Xabier Contreras, Andreas Max Ernst, Felix Wieland, et al.

Dynamic Transbilayer Lipid Asymmetry Gerrit van Meer

For additional articles in this collection, see http://cshperspectives.cshlp.org/cgi/collection/

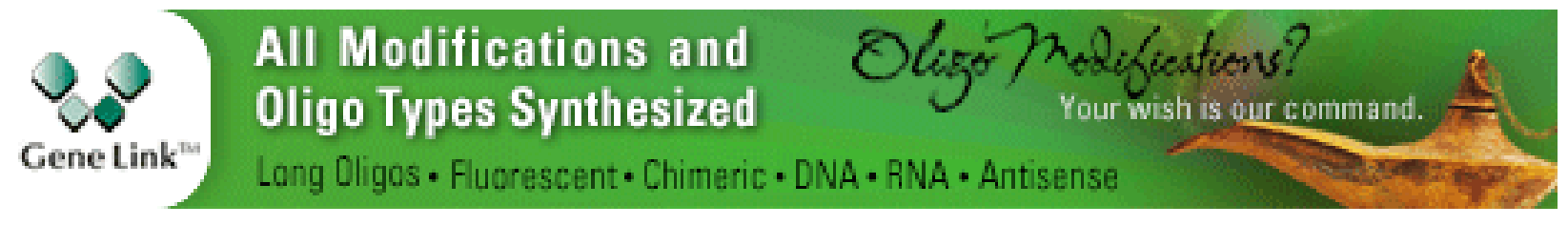

Copyright @ 2011 Cold Spring Harbor Laboratory Press; all rights reserved 